\title{
Benign infantile convulsion as a diagnostic clue of paroxysmal kinesigenic dyskinesia: a case series
}

Naoya Matsumoto, Satoru Takahashi", Akie Okayama, Akiko Araki and Hiroshi Azuma

\begin{abstract}
Introduction: Paroxysmal kinesigenic dyskinesia is characterized by sudden attacks of involuntary movements. It is often misdiagnosed clinically as psychogenic illness, which distresses the patients to a great extent. A correct diagnosis will improve the quality of life in patients with paroxysmal kinesigenic dyskinesia because treatment with low doses of anticonvulsants is effective for eliminating the clinical manifestations. Paroxysmal kinesigenic dyskinesia can occur independently of or concurrently with benign infantile convulsion. Identification of PRRT2 as the causative gene of benign infantile convulsion and paroxysmal kinesigenic dyskinesia allows genetic confirmation of the clinical diagnosis.
\end{abstract}

Case presentation: We describe the clinical features of a Japanese family with either paroxysmal kinesigenic dyskinesia or benign infantile convulsion. A PRRT2 missense mutation (c.981C > G, p.lle327Met) was identified in two patients with benign infantile convulsion and three patients with paroxysmal kinesigenic dyskinesia as well as in two unaffected individuals. Allowing incomplete penetrance in the mutation carriers, this mutation co-segregated completely with the phenotype. The patients with paroxysmal kinesigenic dyskinesia had been misdiagnosed with psychogenic illness for many years. They were correctly diagnosed with paroxysmal kinesigenic dyskinesia when their children visited a pediatrician for benign infantile convulsion. Treatment with carbamazepine controlled their involuntary movements completely.

Conclusions: Paroxysmal kinesigenic dyskinesia is a treatable movement disorder that is often misdiagnosed clinically as psychogenic illness. It is important to note that two clinically distinct disorders, benign infantile convulsion and paroxysmal kinesigenic dyskinesia, are allelic conditions caused by PRRT2 mutations. Paroxysmal kinesigenic dyskinesia should be suspected in families with a child with benign infantile convulsion.

Keywords: Benign infantile convulsion, Mutation, Paroxysmal kinesigenic dyskinesia, PRRT2, Seizures

\section{Introduction}

Paroxysmal dyskinesias are episodic movement disorders characterized by sudden attacks of involuntary movements, such as dystonia, choreoathetosis, and ballism. Most patients are neurologically normal between the attacks and remain conscious through these attacks. On the basis of the events that trigger the abnormal movements, paroxysmal dyskinesias are subdivided into paroxysmal kinesigenic dyskinesia (PKD), paroxysmal exercise-induced dyskinesia (PED), and paroxysmal non-

\footnotetext{
* Correspondence: satoru5p@asahikawa-med.ac.jp

Department of Pediatrics, Asahikawa Medical University, 2-1-1-1

Midorigaoka-Higashi, Asahikawa, Hokkaido 078-8510, Japan
}

kinesigenic dyskinesia (PNKD) $[1,2]$. In patients with $\mathrm{PKD}$, the attacks of abnormal movements are triggered by sudden voluntary movements and last less than 1 minute [3]. The attacks from PED are caused by prolonged exercise and disappear after cessation of physical exercise [4]. PED attacks last between 5 to 30 minutes. By contrast, in patients with PNKD, the attacks occur spontaneously but may be exacerbated by alcohol or caffeine consumption, emotional stress, and fatigue [5]. PNKD attacks last minutes to hours and are typically longer than PKD attacks. Most cases of PKD are familial and inherited in an autosomal dominant trait.

PRRT2 on chromosome 16 (16p11.2), which encodes the Proline-rich transmembrane protein 2 (PRRT2), has 
been identified as the cause of the disease, although its precise role in PKD remains unclear [6]. PKD can occur independently of or concurrently with benign infantile convulsion (BIC) that is characterized by nonfebrile convulsions with onset between 3 and 12 months of age and favorable outcome with normal psychomotor development [7]. Typical seizures are focal with or without secondary generalization and usually occur in clusters.

The diagnosis of PKD is based on the clinical features and the correct diagnosis has implications for treatment and prognosis. However, PKD is often misdiagnosed clinically as epilepsy or psychogenic illness. Recent genetic discoveries will increase the chances of a correct diagnosis and contribute to elucidating the pathophysiology of this disease. Here we report the clinical features of familial cases with either BIC or PKD. The PRRT2 mutation identified in the present study has been reported in a patient with BIC without PKD [8].

\section{Case presentation}

Nine individuals of a Japanese family spanning three generations participated in the study. A detailed family history was obtained by interviewing each family member. Five members had either BIC (Patients 1 and 2, as described below) or PKD (Patients 3, 4, and 5) (Figure 1A). No participant had both PKD and BIC.

Patient 1 (III-2): a boy, now aged 7 years, experienced three afebrile seizures within 1 week at the age of 7 months. The seizures were characterized by a loss of consciousness and hypotonia and lasted from 1 to $3 \mathrm{mi}-$ nutes. His growth and developmental milestones were normal. Interictal electroencephalography (EEG) and cranial computed tomography $(\mathrm{CT})$ revealed normal findings. He was treated with carbamazepine until 2 years of age, and the seizures disappeared and did not reoccur after cessation of the treatment. He has not shown any abnormal paroxysmal movements so far.

Patient 2 (III-3): a boy, now aged 5 years and the younger brother of Patient 1, presented with generalized tonic-clonic seizures that occurred in clusters at 7 months of age. He had normal neurological development. Interictal EEG and cranial CT findings were normal. He was started on carbamazepine and achieved complete seizure control. After cessation of treatment at 2 years of age, he has experienced neither seizure recurrences nor paroxysmal dyskinesias.

Patient 3 (II-2): a woman who had no history of infantile convulsions. At 12 years of age, she presented with abnormal paroxysmal movements occurring several times a day. These episodes were triggered by sudden voluntary movement, such as standing up from a chair, or by emotional stress, for example, when she was asked to write on the blackboard at school. The involuntary movements consisted of choreoathetosis and dystonia

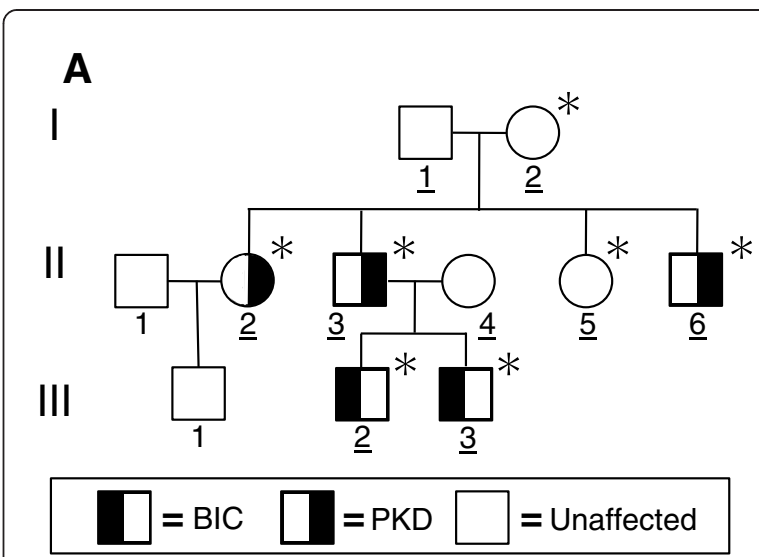

B
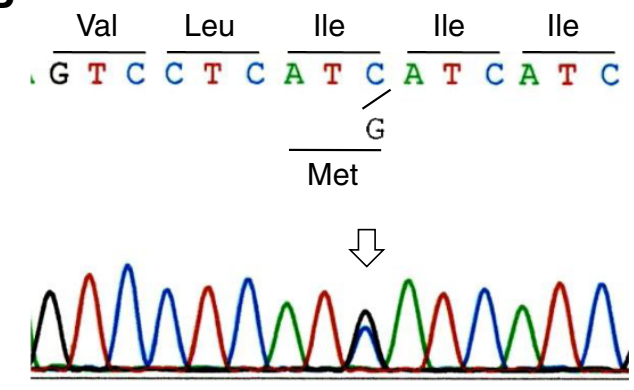

C

M I-1 I-2 II-2 II-3 II-4 II-5 II-6 III-2 III-3

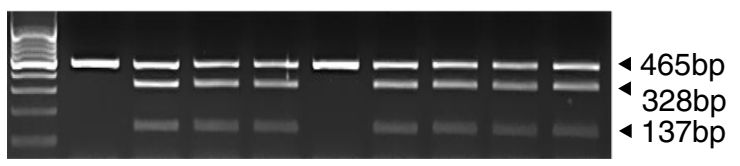

Figure $1 \mathrm{~A}$ familial case with benign infantile convulsion and paroxysmal kinesigenic dyskinesia and PRRT2 mutation. A: Affection status for benign infantile convulsion and paroxysmal kinesigenic dyskinesia are as noted. Underlined pedigree numbers denote individuals whose deoxyribonucleic acid (DNA) was available and who were analyzed in the present study. Asterisks denote PRRT2 mutation carriers. B: Automated DNA sequencing using the polymerase chain reaction product from the patients with either benign infantile convulsion or paroxysmal kinesigenic dyskinesia showed a C-to-G transition at nucleotide 981 in exon 3 of PRRT2 (NCBI accession NM_145239.2), as indicated by the arrow, which resulted in an isoleucine-to-methionine substitution at amino acid position 327 (p.lle327Met). C. Bcll digestion of the exon 3 polymerase chain reaction product showed additional fragments (328bp and 137bp) in two members with benign infantile convulsion (III-2 and III-3) and three members with paroxysmal kinesigenic dyskinesia (II-2, II-3, and II-6) as well as in two unaffected members (I-2 and II-5), which resulted from a C-to-G transition creating a new Bcll restriction site. These additional fragments were observed with the wild-type fragment (465bp), confirming the heterozygous mutation. Abbreviations: BIC, benign infantile convulsion; paroxysmal kinesigenic dyskinesia, PKD; bp, base pairs; Val, valine; Leu, leucine; lle, isoleucine; Met, methionine. 
and lasted less than a minute in duration. She remained conscious during the episodes of involuntary movements. Unfortunately, she was misdiagnosed as having psychogenic illness. She had been distressed for many years until a physician made a correct diagnosis of PKD at 25 years of age. Treatment with carbamazepine controlled her involuntary movements completely.

Patient 4 (II-3): the father of the patients with BIC (III-2 and III-3) had no seizures in his infancy. He displayed intermittent involuntary movements from the age of 10 years. The nature of the movement was dystonic. The attacks were usually triggered by sudden movements or intention to move; for example, when he was going to take an elevator. The severity of attacks varied from episodes severe enough to cause him to fall down to mild episodes that were barely noticed by his friends and relatives. The attacks were never associated with an alteration in his level of consciousness. He had been bothered with involuntary movements for many years. Finally, he was diagnosed with PKD when his child (III-3) visited a pediatrician for BIC. His symptoms disappeared following the start of treatment with carbamazepine.

Patient 5 (II-6): an intellectually disabled man, now aged 24 years, who had a history of perinatal asphyxia and surgically repaired tetralogy of Fallot. He presented with generalized tonic-clonic seizures at 6 years of age. Although a detailed clinical description of his seizures was not available, his seizures were under control with phenobarbital and phenytoin. Family members have occasionally witnessed his dystonic attacks when he forgot to take medicine. The attacks seemed to be provoked by sudden voluntary movements and occurred independently of his habitual seizures. He remained conscious throughout the dystonic attacks.

After obtaining written informed consent from the participants, genomic deoxyribonucleic acid (DNA) was extracted from the peripheral blood leukocytes of each participant and used as the template for polymerase chain reaction (PCR). The compatible primers were used to yield DNA fragments spanning the entire coding region and intron-exon boundaries of PRRT2. The PCR fragments were analyzed using automated sequencing. The mutation identified by direct DNA sequencing was further confirmed by restriction fragment length polymorphism analysis. We identified a PRRT2 missense mutation (c.981C > G, p.Ile327Met) in two participants with BIC and three participants with PKD in this family but also in two unaffected members (Figure $1 \mathrm{~B}$ and $1 \mathrm{C}$ ).

\section{Discussion}

We report a familial case with a heterozygous mutation in PRRT2 that has been identified as the causative gene of PKD. In this family, five individuals had either BIC or PKD. Although BIC and PKD can occur either alone or together, no individual had both BIC and PKD. The PRRT2 missense mutation (c.981C > G, p.Ile327Met) was identified in two patients with BIC and three patients with PKD as well as in two unaffected individuals. The same PRRT2 mutation has been identified in a patient with BIC without PKD [8]. Allowing incomplete penetrance in the mutation carriers, this mutation co-segregated completely with the phenotype. Our results confirmed that two clinically distinct disorders, BIC and PKD, might be allelic conditions caused by PRRT2 mutation. Taking the temporal changes in the expression of the disease into consideration, we could not exclude the possibility that the younger patients with BIC, now aged 5 and 7 years, may develop dyskinesia later in life.

Attacks of PKD and epileptic seizures have several characteristics in common; both show paroxysmal motor manifestations and good response to anticonvulsants. On the basis of neurophysiological and imaging studies, a subcortical origin related to basal ganglia is proposed as being involved in the PKD pathogenesis [9-11], whereas BIC is relevant to cortical origin [12]. However, the co-occurrence of BIC and PKD in a family with a PRRT2 mutation suggests that a subset of epileptic seizures may share a common pathogenic mechanism with PKD. PRRT2 is expressed in brain regions, including the cerebral cortex and basal ganglia, which were implicated in BIC and PKD, respectively [13]. PRRT2 has been shown to interact with the synaptosomal-associated protein of $25 \mathrm{kDa}$ (SNAP25), a presynaptic membrane protein involved in the synaptic vesicle membrane docking and fusion pathway [14]. Thus, interaction of PRRT2 with SNAP25 may play a pivotal role in the regulation of neurotransmitter release [15]. Such a disturbance of synaptic function may be the common pathogenic mechanism underlying motor manifestations in BIC and PKD. The mechanism of age-dependent occurrence and remission of clinical manifestations in BIC and PKD remains to be elucidated.

\section{Conclusions}

Identification of PRRT2 as the causative gene of BIC and PKD allows genetic confirmation of the clinical diagnosis as well as genetic counseling to family members. PKD is often misdiagnosed clinically as epilepsy or psychogenic illness, which distresses the patients to a great extent. PKD should be suspected in families with a child with BIC. A correct diagnosis will improve the quality of life in patients with PKD because treatment with low doses of anticonvulsants, such as carbamazepine and phenytoin, is effective for eliminating the clinical manifestations.

\section{Consent}

This study was approved by the Ethics Committee of Asahikawa Medical University (Approval number: 1043; 
Title: Elucidation of the molecular genetic basis of benign infantile convulsion and paroxysmal kinesigenic choreoathetosis). Written informed consent for the collection of peripheral blood samples and subsequent analyses was obtained from all participants, with the parents giving consent for themselves and on behalf of their children. Concurrently, written informed consent was obtained from the participants for publication of this case report and any accompanying images. A copy of the written consent is available for review by the Editor-inChief of this journal.

\section{Abbreviations}

BIC: benign infantile convulsion; $C T$ : computed tomography; EEG: electroencephalography; PCR: polymerase chain reaction; PED: paroxysmal exercise-induced dyskinesia; PKD: paroxysmal kinesigenic dyskinesia; PNKD: paroxysmal non-kinesigenic dyskinesia; PRRT2: Proline-rich transmembrane protein 2; SNAP25: synaptosomal-associated protein of $25 \mathrm{kDa}$.

\section{Competing interests}

The authors declare that they have no competing interests.

\section{Authors' contributions}

NM carried out the molecular genetic studies and drafted the manuscript; ST identified the patients, carried out the clinical characterizations, and drafted the manuscript; $A O$ and AA conceived the study and participated in its design; HA participated in the design of the study and helped to draft the manuscript; all authors read and approved the final manuscript.

\section{Acknowledgements}

We thank the family members described here, whose help and participation made this work possible. This work was supported in part by Grant-in-Aid for Scientific Research C from the Japan Society for the Promotion of Science (\#22591118)

Received: 21 January 2014 Accepted: 17 March 2014

Published: 1 June 2014

\section{References}

1. Mehta SH, Morgan JC, Seithi KD: Paroxysmal dyskinesias. Curr Treat Options Neurol 2009, 13:170-178.

2. Bhatia KP: Paroxysmal dyskinesias. Mov Disord 2011, 26:1157-1165.

3. Bruno MK, Hallett M, Gwinn-Hardy K, Sorensen B, Considine E, Tucker S, Lynch DR, Mathews KD, Swoboda KJ, Harris J, Soong BW, Ashizawa T, Jankovic J, Renner D, Fu YH, Ptacek LJ: Clinical evaluation of idiopathic paroxysmal kinesigenic dyskinesia: new diagnostic criteria. Neurology 2004, 63:2280-2287.

4. Bhatia KP, Soland VL, Bhatt MH, Quinn NP, Marsden CD: Paroxysmal exercise-induced dystonia: eight new sporadic cases and a review of the literature. Mov Disord 1997, 12:1007-1012.

5. Bruno MK, Lee HY, Auburger GW, Friedman A, Nielsen JE, Lang AE, Bertini E, Van Bogaert P, Averyanov Y, Hallett M, Gwinn-Hardy K, Sorenson B, Pandolfo M, Kwiecinski H, Servidei S, Fu YH, Ptácek L: Genotype-phenotype correlation of paroxysmal nonkinesigenic dyskinesia. Neurology 2007, 68:1782-1789.

6. Chen WJ, Lin Y, Xiong ZQ, Wei W, Ni W, Tan GH, Guo SL, He J, Chen YF, Zhang QJ, Li HF, Lin Y, Murong SX, Xu J, Wang N, Wu ZY: Exome sequencing identifies truncating mutations in PRRT2 that cause paroxysmal kinesigenic dyskinesia. Nat Genet 2011, 43:1252-1255.

7. Szepetowski P, Rochette J, Berquin P, Piussan C, Lathrop GM, Monaco AP: Familial infantile convulsions and paroxysmal choreoathetosis: a new neurological syndrome linked to the pericentromeric region of human chromosome 16. Am J Hum Genet 1997, 61:889-898.

8. Okumura A, Shimojima K, Kubota T, Abe S, Yamashita S, Imai K, Okanishi T, Enoki H, Fukasawa T, Tanabe T, Dibbens LM, Shimizu T, Yamamoto T: PRRT2 mutation in Japanese children with benign infantile epilepsy. Brain Dev 2013, 35:641-646.
9. Ko CH, Kong CK, Ngai WT, Ma KM: Ictal ${ }^{99 m}$ Tc ECD SPECT in paroxysmal kinesigenic choreoathetosis. Pediatr Neurol 2001, 24:225-227.

10. Volonté MA, Perani D, Lanzi R, Poggi A, Anchisi D, Balini A, Comi G, Fazio F: Regression of ventral striatum hypometabolism after calcium/calcitriol therapy in paroxysmal kinesigenic choreoathetosis due to idiopathic primary hypoparathyroidism. J Neurol Neurosurg Psychiatry 2001, 71:691-695.

11. Shirane S, Sasaki M, Kogure D, Matsuda H, Hashimoto T: Increased ictal perfusion of the thalamus in paroxysmal kinesigenic dyskinesia. J Neurol Neurosurg Psychiatry 2001, 71:408-410.

12. Specchio N, Vigevano F: The spectrum of benign infantile seizures. Epilepsy Res 2006, 70S:S156-S167.

13. Heron SE, Grinton BE, Kivity S, Afawi Z, Zuberi SM, Hughes JN, Pridmore C, Hodgson BL, lona X, Sadleir LG, Pelekanos J, Herlenius E, Goldberg-Stern H, Bassan H, Haan E, Korczyn AD, Gardner AE, Corbett MA, Gécz J, Thomas PQ, Mulley JC, Berkovic SF, Scheffer IE, Dibbens LM: PRRT2 mutations cause benign familial infantile epilepsy and infantile convulsions with choreoathetosis syndrome. Am J Hum Genet 2012, 90:152-160.

14. Lee HY, Huang Y, Bruneau N, Roll P, Roberson ED, Hermann M, Quinn E, Maas J, Edwards R, Ashizawa T, Baykan B, Bhatia K, Bressman S, Bruno MK, Brunt ER, Caraballo R, Echenne B, Fejerman N, Frucht S, Gurnett CA, Hirsch E, Houlden H, Jankovic J, Lee WL, Lynch DR, Mohammed S, Müller U, Nespeca MP, Renner D, Rochette J, et al: Mutations in the novel protein PRRT2 cause paroxysmal kinesigenic dyskinesia with infantile convulsions. Cell Rep 2012, 1:2-12.

15. Graham ME, Washbourne P, Wilson MC, Burgoyne RD: Molecular analysis of SNAP-25 function in exocytosis. Ann N Y Acad Sci 2002, 971:210-221.

doi:10.1186/1752-1947-8-174

Cite this article as: Matsumoto et al:: Benign infantile convulsion as a diagnostic clue of paroxysmal kinesigenic dyskinesia: a case series. Journal of Medical Case Reports 2014 8:174.

\section{Submit your next manuscript to BioMed Central and take full advantage of:}

- Convenient online submission

- Thorough peer review

- No space constraints or color figure charges

- Immediate publication on acceptance

- Inclusion in PubMed, CAS, Scopus and Google Scholar

- Research which is freely available for redistribution 